\title{
A SINGLE LOOP PULSATING HEAT PIPE IN VARYING GRAVITY CONDITIONS: EXPERIMENTAL RESULTS AND NUMERICAL SIMULATIONS

\author{
L. Pietrasanta ${ }^{1 *}$, D. Mangini $i^{*}$, D. Fioriti ${ }^{2}$, N. Miche ${ }^{1}$, M. Andredaki, A. Georgoulas, L. Araneo ${ }^{3}, M$. \\ Marengo ${ }^{1}$
}

\author{
${ }^{1}$ School of Computing, Engineering and Mathematics, University of Brighton, Brighton, UK \\ ${ }^{2}$ DESTEC, Universita' di Pisa, Pisa, Italy \\ ${ }^{3}$ Energy Department, Viale Lambruschini 2, Politecnico di Milano, Milan, Italy
}

\begin{abstract}
The thermo-fluid dynamic behaviour of a Single Loop Pulsating Heat Pipe (SLPHP) has been characterized during the $66^{\text {th }}$ ESA Parabolic. The SLPHP, with a $2 \mathrm{~mm}$ inner diameter, has been tested in bottom heated mode, varying the working fluid (FC-72 and ethanol), the heat power input (from $1 \mathrm{~W}$ to $24 \mathrm{~W})$ and the gravity level $(0.01 \mathrm{~g}, 1 \mathrm{~g}$ and $1.8 \mathrm{~g})$. Two transparent tubes connect the evaporator and the condenser, allowing local fluid flow visualization. A set of three-dimensional maps, derived from semi-empirical correlations usually adopted in literature to estimate the critical diameter at different gravity levels are drawn for the different fluids tested, liquid velocities and fluid temperatures. These maps are used to compare the flow velocity observed experimentally with the critical diameter value calculated. Additionally, an enhanced Volume of Fluid (VOF) model is utilised to simulate an imposed slug flow within a straight $2 \mathrm{~mm}$ inner diameter channel, replicating the same experimental conditions, with the primary aim to study the effect of the vapour bubble length and the liquid film thickness on the generated elongated bubble dynamics, in microgravity conditions.
\end{abstract}

KEY WORDS: Two-phase flow, Heat pipe, Boiling and evaporation, microgravity experiments, VOF simulations

\section{INTRODUCTION}

Flow patterns in vapour-liquid flow in micro/milli-channels has been object of many studies in the last two decades [1][2][3] since many important applications, such as miniature heat exchangers for ground and space applications, require a significant reduction in dimensions and weight, coupled with a constant increasing thermal capability. Taylor flow is a dominant pattern during vapour-liquid flow in capillary channels[4]. This flow pattern ensures a reduction in the equivalent thermal resistance value compared to other flow patterns established in a thermally induced, two-phase device better known as Pulsating Heat Pipe (PHP). PHPs local hydrodynamic characteristics such as liquid bulk velocities/accelerations, bubble lengths, pressure drop along the flow, are necessary to understand what causes the slug-plug conditions to degrade in other flow pattern regimes and make a step towards the prediction of local heat transfer coefficients to quantify the effects of these transitions and assist the mathematical and numerical modelling. Many of the works available in literature investigate flow boiling in a single straight channel, with constant imposed mass flow rate[5][6]. These conditions are far from the oscillating flow in a bended PHP serpentine, where the thermo-fluid dynamic behaviour is even more chaotic, being affected for instance also by vapor bubble coalescence, separation, flow reversal and flow pattern transitions. This results in a lack of comprehensive mathematical models able to predict the transport phenomena governing the heat transfer, especially the role of pressure drop [7]. In this paper, an extensive discussion on regime transition for a given channel based on the correlations available in the literature is provided. The concept of a critical velocity that quantitatively marks Taylor flow transitions to other regimes is introduced. A simplified Single Loop Pulsating Heat Pipe (SLPHP) test cell has been designed and tested during the $66^{\text {th }}$ ESA Parabolic Flight Campaign with the purpose 
of investigating the hydrodynamics behind the transitions of flow pattern in a PHP. The bubble velocity of the two-phase flow is estimated from high-speed imaging acquired during experiments via a multipleposition interface-detection velocimetry method and plotted along with temperature, power, gravity field and high-accuracy pressure data for two different fluids (FC-72 and ethanol). An enhanced Volume Of Fluid (VOF) model, previously validated [6]-[8], has been utilised. The effect of vapour slug length, velocity and acceleration on the resulting elongated vapour slug dynamics, under imposed constant pressure drops have been investigated, focusing on isolated vapour slugs within the considered mini-channel, in order to better understand the conditions that lead to a brake-up event. It has to be mentioned that the constant pressure drops are suddenly applied in an initially stagnant two-phase flow domain in order to capture the effect of the flow acceleration and give further insight in the proposed SLPHP experiments.

\section{SLUG-PLUG FLOW CONDITIONS AND REGIMES TRANSITION IN A SINGLE LOOP PHP}

The interaction between gravitational, interfacial, inertial and viscous forces defines the form of a twophase flow in micro-channels. Referring to non-dimensional numbers allows to understand the role of this forces. When surface tension forces dominate gravitational forces, a channel is conventionally defined small and a stratified flow is not anymore observed. A critical Bo number value of 4 [9] has been derived by balancing surface tension and gravitational forces and has been accepted in the PHP community as transition threshold between slug/plug flow to stratified flow typical of thermosyphons, only referring to static conditions obtained in a channel, without considering inertial effects. The corresponding critical diameter is pointed out in (1):

$$
d_{\text {crit }}(B o) \leq 2 \sqrt{\sigma B o_{\text {crit }} / g\left(\rho_{l}-\rho_{v}\right)}
$$

Weber's (We) and Garimella's (Ga) numbers make a step forward compared to Bo number, since both consider inertial effects. Inertial effects play an important role on the flow confinement and flow pattern, in the sense that when the velocity is high, the menisci are unstable and a possible rupture of the vapor bubble can be achieved, as already pointed out [10]. The corresponding critical diameter is given by (2) and (3).

$$
\begin{gathered}
d_{\text {crit }}(W e) \leq 4 \sigma / \rho_{l} u_{l}^{2} \\
d_{\text {crit }}(G a) \leq \sqrt{160 \mu_{l} / \rho_{l} v_{l} \sqrt{\sigma / g\left(\rho_{l}-\rho_{v}\right)}}
\end{gathered}
$$

For the same pair of values of temperature and estimated average velocity, the information on the critical diameter given by the We criterion (Figure 1a) is extremely high for the lowest liquid bulk velocity compared to the value returned by Ga criterion (Figure 1b), because the We criterion does not take into account the viscosity of the fluid and does not provide any information in case of varying gravity field.

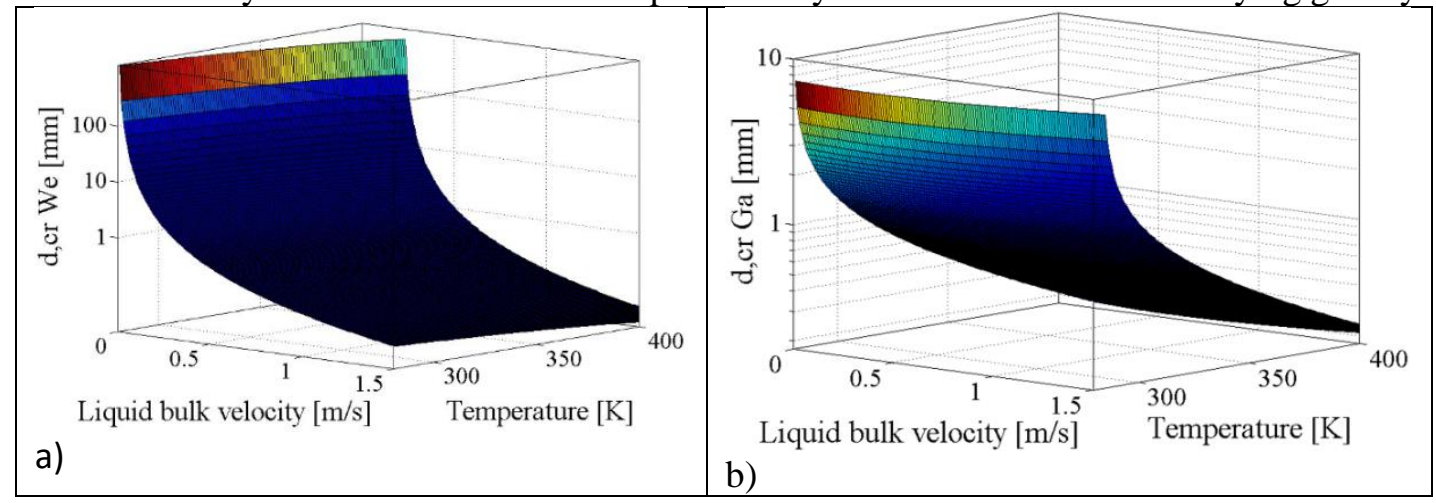

Figure 1 a) Map of critical diameter according to Weber number criterion and b) Garimella number criterion for Ethanol at Earth gravity level. 
Garimella's critical diameter equation has been formulated correlating experimental data obtained at Earth gravity conditions and keeping constant mass flow rate, flow boiling conditions and never validate against very specific experimental data on a PHP device, operating under varying gravity level. Given the continuously varying conditions (velocity, temperature, acceleration, gravity field) inside the channels of a PHP designed for space applications, the idea of a static critical diameter might not be the most appropriate for predicting the flow distribution. Since the inertial force plays an important role on the menisci rupture and the subsequent vapor bubble break-up during PHP operation, a critical velocity (4), derived for a given diameter, has been extracted from Garimella's critical diameter equation to define instantaneous flow pattern:

$$
v_{\text {crit }}(G a)=160 \frac{\mu_{l}}{\rho_{l} d^{2}} \sqrt{\sigma / g\left(\rho_{l}-\rho_{v}\right)}
$$

\section{EXPERIMENTAL APPARATUS AND PROCEDURE}

The test cell, consisting of a closed loop with four 90 degree turns, has been designed to facilitate high speed imaging and high-accuracy, high-frequency pressure investigation coupled with temperature measurement. The main components of the system are shown in Figure 2 . The evaporator and the condenser are made of copper tubes (I.D./O.D. $2 \mathrm{~mm} / 4 \mathrm{~mm}$ ) and are connected by means of two sapphire tubes $(110 \mathrm{~mm}$ axial length, same I.D./O.D. of the copper tubes). Twelve T-type thermocouples $(10 \mathrm{~Hz}$ acq. freq.) that monitor the external wall temperature are placed both in the heated and in the cooled section. Two high-accuracy pressure transducers ( 1 bar absolute, accuracy $0.05 \% \mathrm{FSO}, 100 \mathrm{~Hz}$ acq. freq.), mounted at both ends of one of the sapphire insert. The high-speed camera (resolution 1280x1024 pixel) records images of both the channels at $100 \mathrm{fps}$, with perfect synchronisation with the pressure and temperature data. Three electrical heaters are coiled

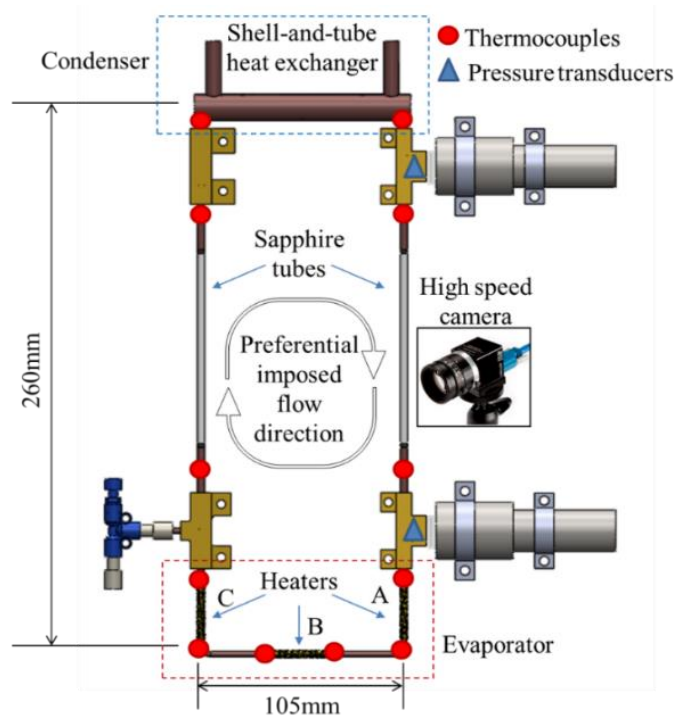

Figure 2 Test cell description around the evaporator in three sections, providing a nominal wall-to-fluid heat flux of $6.5 \mathrm{~W} / \mathrm{cm}^{2}$ at 10 $\mathrm{W}$. The heaters are controlled independently, to vary the heating distribution along the heated zone. An exhaustive description of the test cell can be found in [11]. The independent control of the heating elements allows non-symmetrical heating with respect to the gravity field. As already demonstrated [12]for a similar multi-turn two-phase passive heat transfer device, a non-uniform heating pattern, promotes a circulation in a preferential direction, with a subsequent improvement of the overall thermal performance when the device is gravity assisted. Two working fluid have been tested: Ethanol and FC72. The fluid selection has been based on the static confinement diameter. On ground, FC-72 d,cri $(\mathrm{Bo})$ calculated with eq.(1) is slightly lower than the actual internal diameter, while for ethanol is slightly greater $[10,11]$. Therefore, the effect of the inertial effect on the slug/plug flow breaking can be investigated at different gravity levels. The SLPHP was first evacuated to $0.3 \mathrm{mPa}$ and then partially filled with the selected fluid, with a filling ratio of $0.6 \pm 0.025$, corresponding to a fluid volume of 1.45 $\mathrm{ml}$. After the ground characterization [11], the SLPHP has been tested under varying gravity condition during the ESA $66^{\text {th }}$ Parabolic Flight Campaign. The flight test procedure, in terms of power input to the evaporator, has been reported in Table 1. A detail of power allocated to the three heaters has also been reported, to show the configuration used to impose a preferential direction to the flow. At the occurrence of microgravity (injection phase) of the first parabola of each series, the selected power has been provided to the heaters to investigate the start-up in microgravity conditions. For the other parabolas of the series, the power input has been kept constant throughout the sequence normal gravity - hyper gravity - microgravity - hyper gravity - normal gravity and in the gaps between parabolas. During the breaks between each series $\left(5^{\prime}-5^{\prime}-15^{\prime}-5^{\prime}-5^{\prime}\right)$ the power was switched off completely. 


\subsection{Estimation of the liquid phase velocity}

The velocity of the liquid phase has been evaluated processing high-speed imaging via a multipleposition, liquid phase sensitive, PIV-based velocimetry method. Each frame is segmented into a grid where $\mathrm{I}$ is the number of nodes in the $\mathrm{y}$ direction and $\mathrm{J}$ the number of nodes in the $\mathrm{x}$ direction (Figure 3 ). The spacing of the nodes along the channel has been fixed to 8 pixels after sensitivity analysis. The PIV software allocates a value for each component of velocity ( $\mathrm{x}$ and $\mathrm{y}$ ) to each node and for every frame, reacting to the variation in pixels intensity characteristic of a liquid/vapour interface.

Table 1 Flight test procedure during the ESA 66th PFC

\begin{tabular}{c|c|c|c|c}
\hline Parabola N. & $\begin{array}{c}\text { Ethanol (Day I) and FC-72 } \\
\text { (Day II) Total Power }\end{array}$ & $\begin{array}{c}\text { Heater } \\
\text { A }\end{array}$ & $\begin{array}{c}\text { Heater } \\
\text { B }\end{array}$ & $\begin{array}{c}\text { Heater } \\
\text { C }\end{array}$ \\
\hline Parabola 0 & $15 \mathrm{~W}$ & 0 & 6 & 9 \\
Parabola 1-5 & 9W & 0 & 6 & 3 \\
Parabola 6-10 & $15 \mathrm{~W}$ & 0 & 6 & 9 \\
Parabola 11 & $15 \mathrm{~W}$ & 0 & 6 & 9 \\
Parabola 12-15 & $18 \mathrm{~W}$ & 0 & 6 & 12 \\
Parabola 16 & $18 \mathrm{~W}$ & 0 & 6 & 12 \\
Parabola 17-20 & 24W & 0 & 9 & 15 \\
Parabola 21 & $18 \mathrm{~W}$ & 0 & 6 & 12 \\
Parabola 22-25 & 21W & 0 & 3 & 12 \\
Parabola 26 & 15W & 6 & 9 & 12 \\
Parabola 27-30 & 27W & &
\end{tabular}

In every $\mathrm{J}$ positions, the values of $\mathrm{v}_{\mathrm{x}}$ has been averaged in the radial direction (y), excluding node 1 and node I to avoid wall effects for all the images of the sequence analysed, in order to obtain the temporal trend of the vapor bubble velocities.

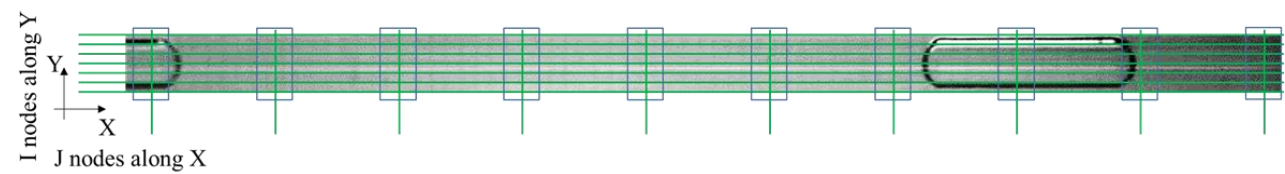

Figure 3 Velocity nodes grid overlapped on a frame. Position of the radial averaging sections.

\section{EXPERIMENT RESULTS DATA ANALYSIS}

\subsection{Start-up in microgravity}

Microgravity start-up and activation were investigated for two different fluids and different heat flux input levels. An estimation of the energy required for causing a pressure perturbation greater than $1 \mathrm{kPa}$, has been calculated as product of the power input and the duration of the heating phase before the occurring of the pressure perturbation (Table 2).

Table 2 Activation energy during the first parabola of each series for Ethanol and FC-72

\begin{tabular}{c|c|c|c|c|c}
\hline \multirow{2}{*}{$\begin{array}{c}\text { Parabola } \\
\text { Number }\end{array}$} & Heat Flux Input & \multicolumn{2}{|c|}{ Microgravity Start-up quality ${ }^{1}$} & \multicolumn{2}{|c}{ Start-up energy [J] } \\
\cline { 3 - 5 } & {$\left[\mathrm{W} / \mathrm{m}^{2}\right]$} & Ethanol & FC-72 & Ethanol & FC-72 \\
\hline 0 & $3.18 \cdot 10^{4}$ & Strong pulsation & Strong pulsation & 220 & 156 \\
6 & $3.18 \cdot 10^{4}$ & No activation & Strong pulsation & - & 166 \\
11 & $3.18 \cdot 10^{4}$ & No activation & Weak pulsation & - & 265 \\
16 & $3.82 \cdot 10^{4}$ & No activation & Weak pulsation & - & 223 \\
21 & $3.82 \cdot 10^{4}$ & No activation & Strong pulsation & - & 161 \\
26 & $3.18 \cdot 10^{4}$ & No activation & Weak pulsation & - & 217 \\
\hline
\end{tabular}

\footnotetext{
${ }^{1}$ Examples of "Strong" pulsation can be found in Figure 4. A pulsation similar to the one obtained with FC-72 at $1.91 \cdot 10^{4} \mathrm{~W} / \mathrm{m}^{2}$, with at least one pressure peak equal or above $1 \mathrm{kPa}$ can be considered strong. Anything below is considered weak. No activation is when no significative pulsation is detected, and the pressure trend is decreasing.
} 
Strong pulsation with FC-72 are recorded just in half of the cases: this may be caused by the favourable distribution of liquid phase in the evaporator zone during the heating when microgravity is reached during the parabolic flight trajectory. As an effect of the pulsation induced in the system, a decrease in the evaporator temperature can be appreciate. FC-72 shows fluid pulsations during all microgravity periods (Figure 4): isolated peaks of pressure are alternating with a stop in the overall motion. The intermittent behaviour in weightlessness is mainly due to the simplified nature of the device, not able to introduce enough thermo-hydrodynamic instabilities when it is not gravity assisted. Comparing the two fluids, the inhibition of any sort of pulsation for Ethanol, seems to be caused mainly by the difference in the latent heat of evaporation $(920 \mathrm{~kJ} / \mathrm{Kg}$ for Ethanol, $88 \mathrm{~kJ} / \mathrm{kg}$ for FC-72 at $300 \mathrm{~K})$ and by the $\Delta P / \Delta T(500 \mathrm{~Pa} / \mathrm{K}$ for Ethanol, $2000 \mathrm{~Pa} / \mathrm{K}$ for $\mathrm{FC}-72$ at $300 \mathrm{~K})$ given the comparable order of magnitude of all other properties.

Figure 4 Microgravity behaviour of different fluids with different heat flux input and effect of pressure oscillations on the evaporator temperature (with relative thermocouples positioning).

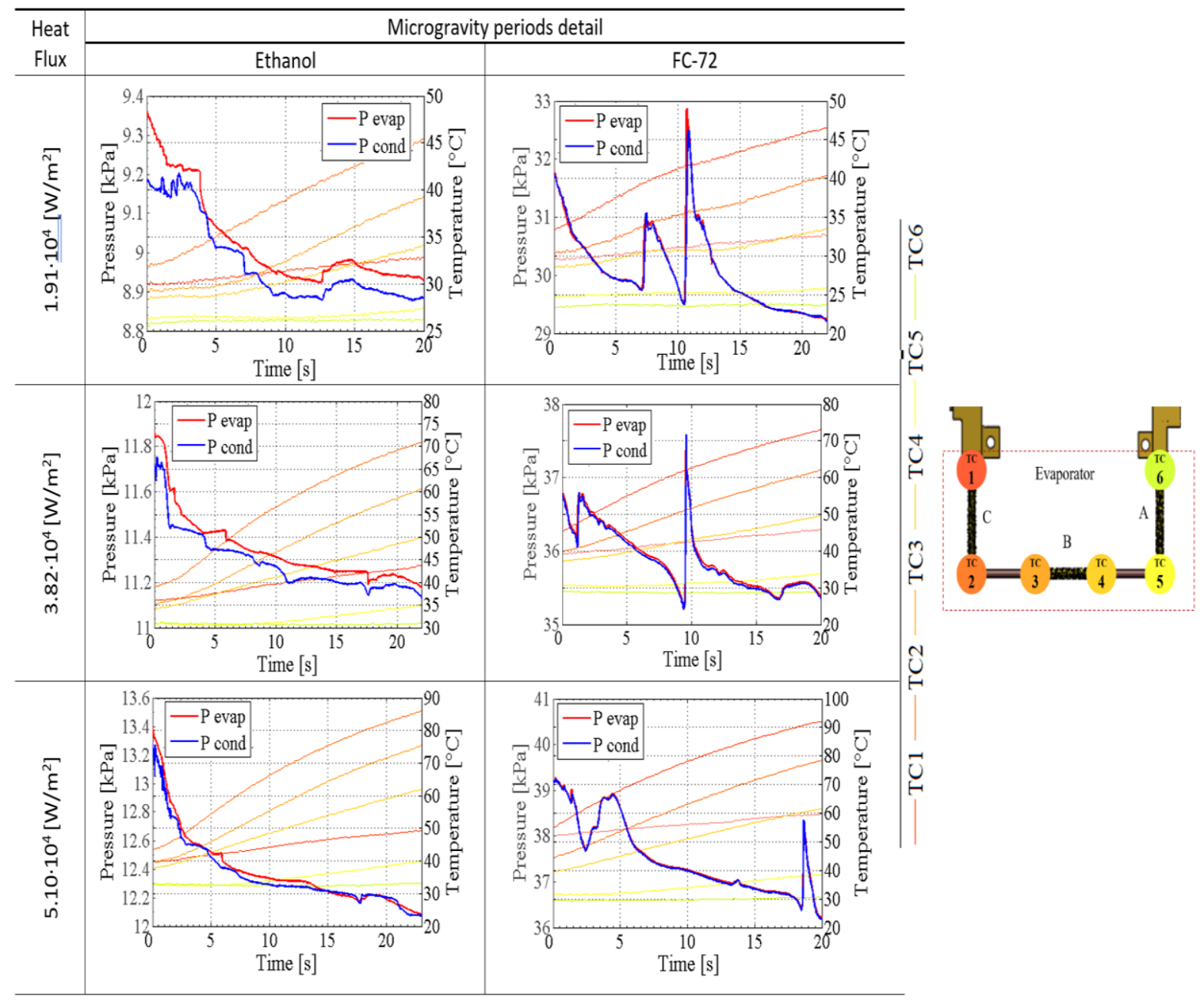

\subsection{Inertial effects on the flow pattern transitions}

Useful information about the constant transition between flow patterns in a PHP can be obtained from the ratio between the velocity estimated at time $i$ and the value of critical velocity, for a given and constant value of temperature:

$$
\phi_{i}=\left.\frac{v_{l, i}}{v_{\text {crit }}}\right|_{T=\text { const }}
$$

Looking at Figure 5, a dynamic capillary quantitative ratio greater than the unit, means a deformation of the slug-plug that can leads to a break-up of the bubble or marks the transition to a semi-annular or annular regime. 


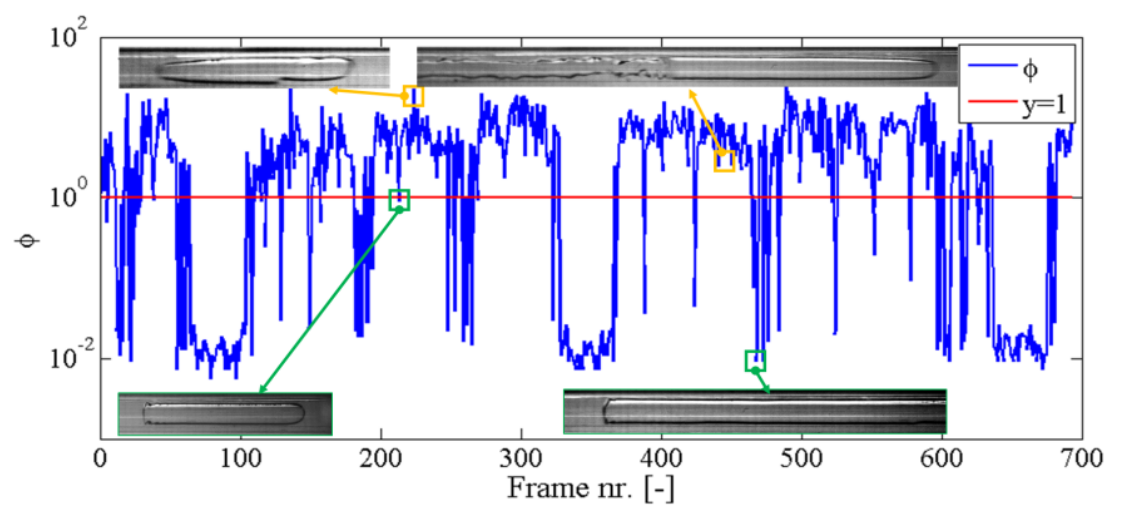

Figure 5 Ratio between estimated velocity and critical velocity for $2 \mathrm{~mm}$ sapphire tube filled with FC72, during 1g period; all properties evaluated at $23^{\circ} \mathrm{C}$.

\section{PARAMETRIC NUMERICAL SIMULATIONS}

In the present section of the current work, a previously developed tested and validated, enhanced Volume Of Fluid (VOF) based method that has been developed in the general context of OpenFOAM CFD Toolbox [11]-[12], is utilised to perform a plethora of parametric numerical simulations aiming to isolate and identify the effect and importance of fundamental controlling parameters in the resulting elongated vapour bubble dynamics within the considered circular mini-channel branch of the SLPHP that has been described in Section 3. Such kind of two-phase CFD simulations have been widely used in the literature so far to investigate elongated vapour bubble dynamics within a previously developed single phase flow under constant pressure drops or constant imposed mass flow rates [13,14]. However, the focus of the present numerical investigation is in cases of instantaneous pressure drops (i.e. accelerations) for small time periods, of approximately $100 \mathrm{~ms}$, that the pressure drop between the condenser and the evaporator has been identified of being constant and the two-phase flow, moves towards a preferential direction. A computational domain that imitates the mini-channel branch between the 2 pressure transducers in the considered SLPHP (Fig. 2), is adopted. Therefore, a circular straight mini-channel of a diameter of $2 \mathrm{~mm}$ and total length of $190 \mathrm{~mm}$ is considered for the present parametric numerical experiments. The proposed computational domain, the computational mesh and the applied boundary conditions are illustrated in Figure 5. As it can be observed, a non-uniform structured computational mesh consisting of a total number of 972,000 cells has been adopted, using gradient refinement in the redial direction towards the channel wall. The proposed computational mesh has been selected after a mesh independency study. For the simulations, a vapour slug of a certain initial length and liquid film thickness is positioned at a distance of $10 \mathrm{~mm}$ from the inlet of channel, within a stagnant liquid domain that fills the rest of the channel. Then a sudden pressure drop is applied between the inlet and the outlet of the channel that generated a liquid flow that accelerates the bubble towards the outlet. All simulations are conducted for a short time period of $100 \mathrm{~ms}$. The details of the overall simulations conducted are summarised in Table 3 . As it can be seen two different series of parametric numerical simulations were performed, with 15 runs in each case. In each series, the effects of the initial bubble length, the initial liquid film thickness and the applied pressure drop on the resulting vapour bubble dynamics is investigated for Ethanol and FC-72, respectively. It should be mentioned that in all of the tested cases, the gravitational acceleration is set to $0 \mathrm{~m} / \mathrm{s}^{2}$. The previously described simulation procedure is followed, in order to investigate the effect of the generated flow acceleration in the elongated bubble dynamics due to a sudden pressure drop, that is of more relevance and interest in order to give further insight into the observed instabilities captured by the high-speed images, in the experiments (e.g. see experimental snapshots in Figure 6). Adiabatic conditions are also assumed, and the working fluid properties in each case are selected as Ethanol liquid-vapour and FC-72 liquid-vapour, at a saturation equilibrium point, that corresponds to a saturation temperature $\mathrm{T}_{\text {sat }}=24.9^{\circ} \mathrm{C}$ for Ethanol and $\mathrm{T}_{\text {sat }}=23^{\circ} \mathrm{C}$ for FC-72, in accordance to the thermocouple readings, in specific selected time periods from the SLPHP experiments, were the temperature as well as the pressure difference between the two pressure transducers were approximately constant. The effects of initial bubble length, initial liquid film thickness and applied pressure drop, are illustrated in Figures 5 and 6 for the cases of Ethanol and FC72 , respectively. In the proposed figures, the instantaneous resulting bubble shape (dark grey colour) 
and its position within the accelerated liquid flow (light grey colour) within the considered minichannel, is depicted.

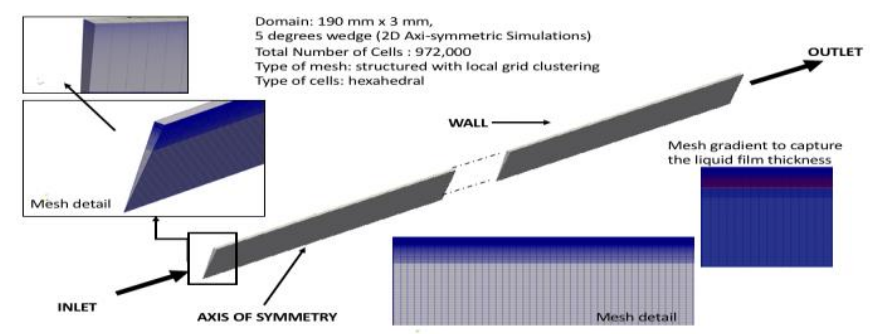

Figure 6 Computational domain, mesh and boundary conditions

Table 3 Parametric numerical simulation framework.

\begin{tabular}{|c|c|c|c|c|c|c|c|c|c|c|c|c|c|c|c|}
\hline Case & 1 & 2 & 3 & 4 & 5 & 6 & 7 & 8 & 9 & 10 & 11 & 12 & 13 & 14 & 15 \\
\hline Fluid & \multicolumn{15}{|c|}{ Ethanol } \\
\hline$\Delta \mathbf{P}[\mathbf{P a}]$ & \multicolumn{3}{|c|}{550} & \multicolumn{3}{|c|}{750} & \multicolumn{3}{|c|}{1100} & & 0 & & & & 00 \\
\hline $\mathbf{L}_{b}[\mathrm{~mm}]$ & 6 & 12 & 18 & 6 & 12 & 18 & 6 & 12 & 18 & \multicolumn{6}{|c|}{12} \\
\hline$\delta[\mu \mathrm{m}]$ & \multicolumn{9}{|c|}{200} & 50 & 100 & 50 & 100 & 50 & 100 \\
\hline Case & 16 & 17 & 18 & 19 & 20 & 21 & 22 & 23 & 24 & 25 & 26 & 27 & 28 & 29 & 30 \\
\hline Fluid & \multicolumn{15}{|c|}{ FC-72 } \\
\hline$\Delta P[\mathrm{~Pa}]$ & \multicolumn{3}{|c|}{550} & \multicolumn{3}{|c|}{750} & \multicolumn{3}{|c|}{1100} & & 0 & & & & 00 \\
\hline $\mathbf{L}_{\mathbf{b}}[\mathbf{m m}]$ & 6 & 12 & 18 & 6 & 12 & 18 & 6 & 12 & 18 & \multicolumn{6}{|c|}{12} \\
\hline$\delta[\mu \mathrm{m}]$ & \multicolumn{9}{|c|}{200} & 50 & 100 & 50 & 100 & 50 & 100 \\
\hline
\end{tabular}

For both working fluids considered, the bubble length significantly affects the resulting liquid film dynamics for the same pressure drop and liquid film thickness. In more detail as the bubble length increases capillary ridges appear in the liquid film between the vapour slug and the wall of the channel. In the case of Ethanol, it is also characteristic that for lower values of initial liquid film thickness the proposed capillary ridges are not present any more. On the other hand, for FC-72 at $\mathrm{t}=85 \mathrm{~ms}$ and for an applied pressure drop of $550 \mathrm{~Pa}$, the resulting vapour slugs for all three liquid film thicknesses tested, disintegrate close to their trailing edge into smaller bubbles. The proposed disintegration is more severe for lower liquid film thicknesses. This disintegration is not present in the case of Ethanol due to the significantly higher surface tension coefficient. For both Ethanol and FC-72 the increase of the applied pressure drop leads to more intense interface instabilities in the vicinity of the trailing edge of the bubbles, that in the case of FC-72 lead once more to bubble disintegration close to the trailing edge.

\section{CONCLUSIONS}

The lack of understanding the local characteristic of a slug flow has limited the modelling of PHPs. In this work, the transition between flow pattern regimes has been investigated experimentally and a CFDbased numerical model has been also utilised to provide further insight in the experimental analysis. Inertial effects play a fundamental role on the flow confinement and flow pattern. The actual velocity of the liquid phase has been compared for the first time with a critical velocity value that, according to the most reliable semi-empirical correlation, marks the transitions between a slug flow and annular/semi-annular regimes. In addition, the activation of the oscillations proper of a PHP device has been investigated, confirming the importance of the latent heat of vaporization of the selected working fluid during the start-up. From the overall numerical simulation results, it is evident that both the bubble length as well as the liquid film thickness significantly affect the resulting liquid film dynamics and the accompanying bubble break-up mechanisms, that can be responsible for flow regime transitions within PHPs. Both of these important parameters are not taken into consideration in the currently utilised criteria for the analysis and classification of experimental data. Therefore, the further revision and modification of the proposed criteria to incorporate the effects of bubble length and liquid film thickness, can lead to even more detailed analysis and classification of two-phase flow regimes in PHPs. 


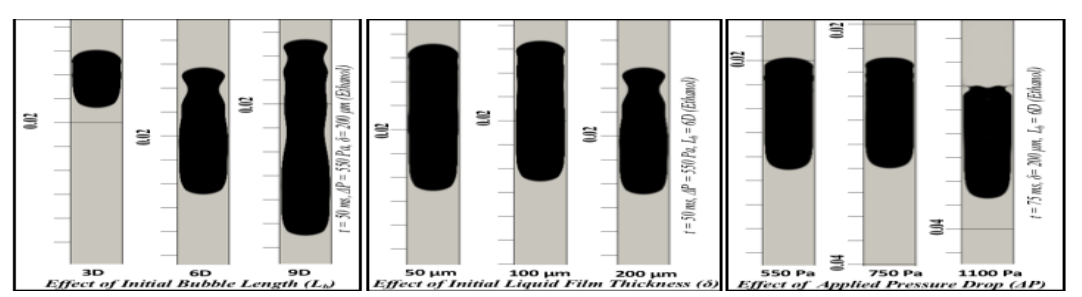

Figure 7 Effects of initial bubble length (left), initial liquid film thickness (middle) and Applied pressure drop (right) on the generated liquid film dynamics, for the case of Ethanol.

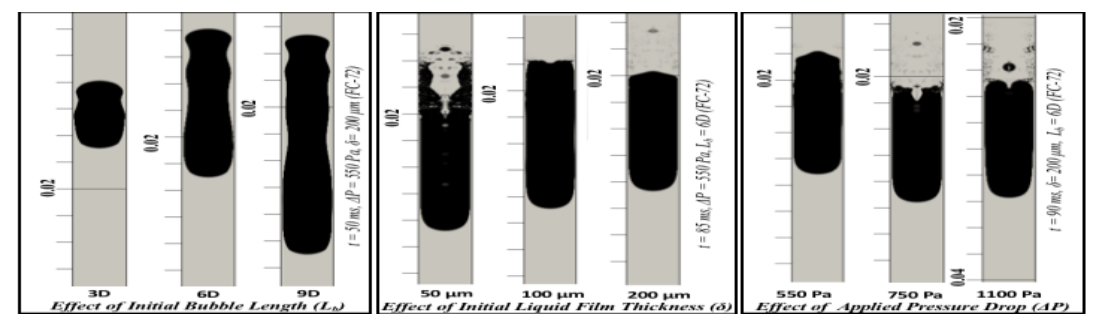

Figure 8 Effects of initial bubble length (left), initial liquid film thickness (middle) and applied pressure drop (right) on the generated liquid film dynamics, for the case of FC-72.

\section{ACKNOWLEDGMENTS}

The Authors would like to acknowledge UK's Engineering and Physical Science Research Council support through the grant EP/P013112/1 as well as the ESA MAP Project INWIP. The authors would also like to acknowledge the contribution of the High-Performance Computing facility of the School of Computing Engineering and Mathematics, in the University of Brighton.

\section{REFERENCES}

[1] R. Gupta, D. F. Fletcher, and B. S. Haynes, "Taylor Flow in Microchannels: A Review of Experimental and Computational Work," J. Comput. Multiph. Flows, vol. 2, no. 1, pp. 1-31, 2010.

[2] L. Chen, Y. Tian, and T. Karayiannis, "The effect of tube diameter on vertical two-phase flow regimes in small tubes," Int. J. Heat Mass Transf., vol. 49, no. 21-22, pp. 4220-4230, Oct. 2006.

[3] T. Chen and S. V. Garimella, "Measurements and high-speed visualizations of flow boiling of a dielectric fluid in a silicon microchannel heat sink," Int. J. Multiph. Flow, vol. 32, no. 8, pp. 957-971, 2006.

[4] P. Angeli and A. Gavriilidis, "Hydrodynamics of Taylor flow in small channels: a review," J. Mech. Eng. Sci., vol. 222, pp. 737-751, 2008.

[5] A. M. Rahatgaonkar and P. R. Pachghare, "Thermo-Hydrodynamics of Taylor Bubble Flow in Context of Pulsating Heat Pipe : a Review,” Int. Res. J. Eng. Technol., vol. 4, no. 6, pp. 1879-1884, 2017.

[6] A. Georgoulas, P. Koukouvinis, M. Gavaises, and M. Marengo, "Numerical investigation of quasi-static bubble growth and detachment from submerged orifices in isothermal liquid pools: The effect of varying fluid properties and gravity levels,” Int. J. Multiph. Flow, vol. 74, pp. 59-78, 2015.

[7] A. Georgoulas, M. Andredaki, and M. Marengo, "An Enhanced VOF Method Coupled with Heat Transfer and Phase Change to Characterise Bubble Detachment in Saturated Pool Boiling," Energies, vol. 10, no. 3, p. 272, Feb. 2017.

[8] E. Teodori, P. Pontes, A. Moita, A. Georgoulas, M. Marengo, and A. Moreira, "Sensible heat transfer during droplet cooling: Experimental and numerical analysis," Energies, vol. 10, no. 6, 2017.

[9] P. A. Kew and K. Cornwell, "Correlations for the prediction of boiling heat transfer in small-diameter channels," Appl. Therm. Eng., vol. 17, no. 8-10, pp. 705-715, 1997.

[10] D. Mangini, M. Mameli, A. Georgoulas, L. Araneo, S. Filippeschi, M. Marengo, "A Pulsating Heat Pipe for Space applications: Ground and Microgravity experiments," Int. J. Therm. Sci., vol. 95, pp. 53-63, 2015.

[11] A. Ilinca, D. Mangini, M. Mameli, D. Fioriti, S. (University of P. Filippeschi, L. Araneo, N. Roth, and M. Marengo, "Fluid-flow pressure measurements and thermo- fluid characterization of a single loop two-phase passive heat transfer device Fluid-flow pressure measurements and thermo-fluid characterization of a single loop two-phase passive heat transfer device," J. Phys. Conf. Ser., 2017.

[12] M. Mameli, D. Mangini, G. F. T. Vanoli, L. Araneo, S. Filippeschi, and M. Marengo, "Advanced multi-evaporator loop thermosyphon," Energy, vol. 112, pp. 562-573, Oct. 2016.

[13] M. Magnini, J. R. Thome, "A CFD study of the parameters influencing heat transfer in microchannel slug plug flow boiling," International Journal of Thermal Sciences, vol. 110, pp. 119-136, 2016.

[14] A. Ferrari, M. Magnini, J. R. Thome, "Numerical analysis of slug flow boiling in square microchannels," International Journal of Heat and Mass Transfer, vol. 123, pp. 928-573, Oct. 2018. 